\title{
THE PICARD GROUP OF A GENERAL TORIC VARIETY OF DIMENSION THREE
}

\author{
T. J. FORD AND R. STIMETS
}

\begin{abstract}
AвstRACT. Let $\Delta$ be a complete fan in $\mathbb{R}^{3}$ such that every three-dimensional cone in $\Delta$ is non-simplicial. In any non-empty open neighborhood of $\Delta$ there is a fan $\Delta^{\prime}$ such that every $\Delta^{\prime}$-linear support function is linear and the Picard group of the associated toric variety is zero.
\end{abstract}

\section{INTRODUCTION}

Let $k$ be a field. Let $N=\mathbb{Z}^{r}$ and denote by $T_{N}$ the $k$-torus on $N$. A fan $\Delta$ is a finite set of strongly convex polyhedral cones in $N \otimes \mathbb{R}=\mathbb{R}^{r}$ such that for all $\sigma, \tau$ in $\Delta$, every face of $\sigma$ is in $\Delta$ and $\sigma \cap \tau$ is a face of both $\sigma$ and $\tau$. If each cone in $\Delta$ is rational, then associated to $\Delta$ is the toric variety over $k$ denoted $X=T_{N} \operatorname{emb}(\Delta)$ [1], [6], [7]. To what extent do the combinatorial properties of the fan $\Delta$ determine the Picard group of $X$ ? This question was addressed in [3] and [5] where many partial solutions are given.

The relation $\sigma \leq \tau$ if $\sigma$ is a face of $\tau$ defines a partial ordering on the set of cones in $\Delta$. Denote by $\Delta_{\text {poset }}$ this partially ordered set. Two fans $\Delta, \Sigma$ are of the same combinatorial type if there is an isomorphism $\Delta_{\text {poset }} \cong \Sigma_{\text {poset }}$ of partially ordered sets.

For example, if $\Delta$ is a simplicial complete rational fan in $\mathbb{R}^{r}$, the Picard group of $X$ is a free $\mathbb{Z}$-module of rank $v-r$ where $v$ is the number of one-dimensional cones in $\Delta$. It follows that for complete simplicial fans, the Picard group of $X$ is determined by the combinatorial type of the fan. For the details, see [6, pp. 63-65] or [5, Theorem 3.2].

To see that the Picard group is not determined by the combinatorial type of the fan, consider another example. The reader is referred to [5, Example 4.6] or [6, pp. 25-26] for the details. If $\Delta$ is the fan over the faces of the cube with vertices at $( \pm 1, \pm 1, \pm 1)$ in $\mathbb{R}^{3}$ and $X$ is the associated toric variety, then the Picard group of $X$ is $\mathbb{Z}$. Let $\Delta^{\prime}$ be the fan with cones spanned by the same sets of generators except that the vertex $(1,1,1)$ is replaced by $(2,1,1)$. If $X^{\prime}$ is the toric variety associated to $\Delta^{\prime}$, then the Picard group of $X^{\prime}$ is zero. The fans $\Delta$ and $\Delta^{\prime}$ are of the same combinatorial type but their Picard groups are not isomorphic. Note that the maximal cones of $\Delta$ are non-simplicial.

Let $\Delta$ be a complete rational fan in $\mathbb{R}^{3}$ whose maximal cones are non-simplicial. The main result of this article, Theorem 1 , proves that there is a rational fan $\Delta^{\prime}$ of the same combinatorial type as $\Delta$ which is "close to $\Delta^{\prime \prime}$ in a way made precise later such that if $X^{\prime}$ is the toric variety associated to $\Delta^{\prime}$, then the Picard group of $X^{\prime}$ is

Date: March 3, 2014.

2000 Mathematics Subject Classification. Primary 14M25; Secondary 14C22.

Key words and phrases. toric variety, Picard group . 
zero. Corollary 1 shows that the fan $\Delta^{\prime}$ can be taken as a general rational point of a real manifold parametrizing a large set of fans of the same combinatorial type as $\Delta$. In particular, it follows that the variety $X^{\prime}$ is non-projective and $\Delta^{\prime}$ is not the fan of cones over the faces of any integral convex polytope containing the origin in its interior. These results were conjectured in [5, §4].

The proof of Theorem 1 uses the fact that the non-maximal cones of $\Delta$ are simplicial. For this reason, the proof does not extend to dimensions greater than three. Nevertheless, it seems plausible that a generalization of Theorem 1 should be true in higher dimensions.

There is a topology on the set of cones in $\Delta$ where the open sets are the subfans of $\Delta$. Denote by $\Delta_{t o p}$ this topological space. Notice that two fans $\Delta, \Sigma$ are of the same combinatorial type if and only if $\Delta_{t o p}$ is homeomorphic to $\Sigma_{t o p}$. The assignment $\Delta \mapsto \Delta_{\text {top }}$ defines a functor $\mathfrak{T}$ from the category of fans on $\mathbb{R}^{r}$ to the category of finite topological spaces.

Let $\Delta(i)=\{\sigma \in \Delta \mid \operatorname{dim}(\sigma)=i\}$. Set $\Delta(1)=\left\{r_{0}, \ldots, r_{n}\right\}$. The intersection of $\Delta(1)$ with the unit sphere $S$ in $\mathbb{R}^{r}$ is a finite set of points, say $\left\{p_{0}, \ldots, p_{n}\right\}$. About each $p_{i}$ we can find an open ball $B_{i}$ on $S$ such that if $p_{i}$ is parametrized by $B_{i}$, then each choice of $\vec{p}=\left(p_{0}, p_{1}, \ldots, p_{n}\right)$ in $B_{0} \times B_{1} \times \cdots \times B_{n}$ defines a fan $\Phi=\Phi(\vec{p})$ such that $\Phi_{\text {top }} \cong \Delta_{\text {top }}$. The manifold $B=\prod_{i=0}^{n} B_{i}$ parametrizes a subset of fans in the fiber $\mathfrak{T}^{-1}\left(\Delta_{\text {top }}\right)$. Call $B$ an open neighborhood of $\Delta$. If $\vec{p} \in B$, then the fan $\Phi=\Phi(\vec{p})$ is not necessarily rational. Sometimes it will be necessary to refer to points in $B$ that give rise to rational fans. In this case let

(1) $B_{\text {rat }}=\left\{\left(p_{0}, \ldots, p_{n}\right) \mid\right.$ for each $i$, $p_{i}$ is the intersection of a rational 1-dimensional cone $r_{i}$ with $\left.B_{i}\right\}$.

If $\sigma$ is a cone in $N \otimes \mathbb{R}$, define $\mathcal{S F}(\sigma)$ to be $\operatorname{Hom}_{\mathbb{R}}(\mathbb{R} \sigma, \mathbb{R})$. The group of $\Delta$-linear support functions, denoted $\mathcal{S} \mathcal{F}(\Delta)$, is by definition the kernel of $\delta^{0}$ in the Čech complex

$$
0 \rightarrow \underset{i}{\oplus} \mathcal{S F}\left(\sigma_{i}\right) \stackrel{\delta^{0}}{\rightarrow} \underset{i<j}{\oplus} \mathcal{S} \mathcal{F}\left(\sigma_{i j}\right)
$$

where $\left\{\sigma_{0}, \ldots, \sigma_{w}\right\}$ is the set of maximal cones of $\Delta$. Set

$$
\kappa=\operatorname{dim}_{\mathbb{R}} \mathcal{S F}(\Delta)
$$

Let $M=\operatorname{Hom}_{\mathbb{Z}}(N, \mathbb{Z})$. There is a natural map $M \otimes \mathbb{R} \rightarrow \mathcal{S F}(\Delta)$. A support function in the image of $M \otimes \mathbb{R}$ is said to be linear.

\section{The MAIN RESUlt}

Theorem 1. Let $\Delta$ be a complete fan on $\mathbb{R}^{3}$ such that every three-dimensional cone in $\Delta$ is non-simplicial. Let $B$ be an open neighborhood of $\Delta$. There is a fan $\Delta^{\prime}$ in $B$ such that every $\Delta^{\prime}$-linear support function is linear. The fan $\Delta^{\prime}$ can be taken to be rational, and if so, the Picard group of the associated toric variety is zero.

Proof. The proof of Theorem 1 is split into a series of lemmas and occupies the remainder of this section. The statement about the Picard group follows from the first claim, by the next lemma. 

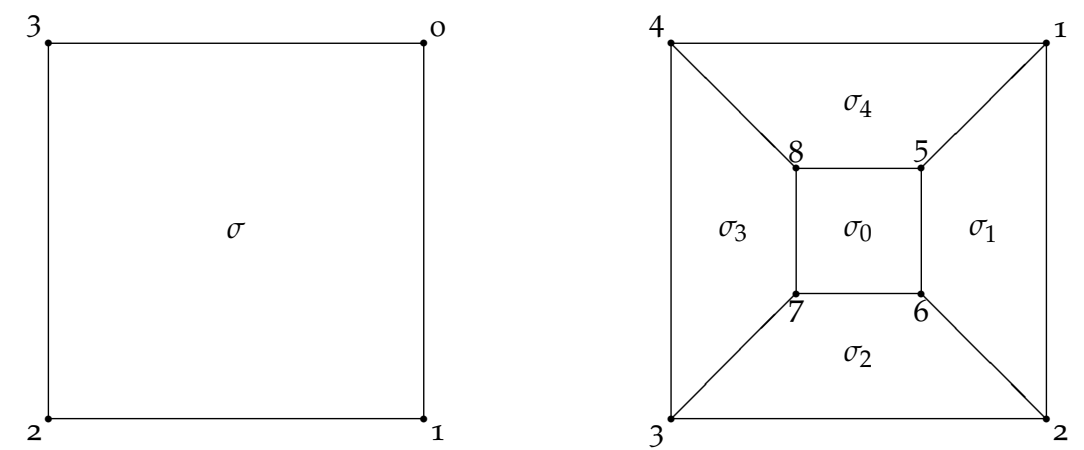

FigURE 1. A cubing of a cone with four edges.

Lemma 2. For a complete rational fan $\Delta$ on $\mathbb{R}^{r}$, if every $\Delta$-linear support function is linear, then the Picard group of $X=T_{N} \operatorname{emb}(\Delta)$ is zero.

Proof. If $\Delta$ is a fan of rational cones and $X=T_{N} \operatorname{emb}(\Delta)$, then it follows from [2, Lemma 8] that there is an exact sequence

(4)

$$
M \otimes \mathbb{R} \rightarrow \mathcal{S F}(\Delta) \rightarrow \operatorname{Pic} X \otimes \mathbb{R} \rightarrow 0 .
$$

In this case, set

$$
\rho=\operatorname{dim}_{\mathbb{R}} \operatorname{Pic} X \otimes \mathbb{R}
$$

If we assume moreover that $\Delta$ is a complete fan, then $X$ is compact and $M \otimes \mathbb{R} \rightarrow$ $\mathcal{S F}(\Delta)$ is an injection. The Picard group of $X$ is a finitely generated torsion free abelian group of rank $\rho$. Combining the above, we have shown $\kappa=\rho+r$ and the lemma follows.

Let $\Delta$ be a fan on $\mathbb{R}^{3}$ which has at least one three-dimensional cone. Let $\sigma \in \Delta(3)$ and suppose $\sigma$ has $n$ one-dimensional faces. Define a cubing of $\sigma$ to be any subdivision of $\sigma$ that inserts $n$ new one-dimensional cones $r_{n+1}, \ldots, r_{2 n}$, $2 n$ new two-dimensional cones and $n+1$ three-dimensional cones $\sigma_{0}, \sigma_{1}, \ldots, \sigma_{n}$. Write cube $(\sigma)$ for a fan which corresponds to a cubing of the cone $\sigma$. This is illustrated in Figure 1 for $n=4$ and Figure 2 for a cone $\sigma$ with $n$ edges. There is a morphism of fans cube $(\sigma) \rightarrow \Delta(\sigma)$. If $\Sigma$ is the subdivision of $\Delta$ corresponding to a cubing of $\sigma$, there is a morphism of fans $\Sigma \rightarrow \Delta$. The natural map $\operatorname{SF}(\Delta) \rightarrow$ $\mathrm{SF}(\Sigma)$ is injective. It was shown in [5, Example 3.6] that for a general cubing of a simplicial three-dimensional cone $\sigma$, a linear support function on cube $(\sigma)$ is linear. The same result was shown in [5, Example 4.6] for the cone $\sigma$ of Figure 1. The next lemma proves this for all $n$.

Lemma 3. Let $\sigma$ be a three dimensional cone in $\mathbb{R}^{3}$ and suppose $\sigma$ has $n$ one-dimensional faces. For a general choice of $r_{n+1}, \ldots, r_{2 n}$ in a cubing of $\sigma$, the fan $\Xi=$ cube $(\sigma)$ has the property that a $\Xi$-linear support function is linear.

Proof. A $\Xi$-linear support function $h$ is determined by its values on the cones of dimension one. The group of $\Xi$-linear support functions can be embedded in $\bigsqcup_{i=1}^{2 n} \mathbb{R} \cdot r_{i}$ as the kernel of a linear transformation defined by a matrix with $2 n$ 


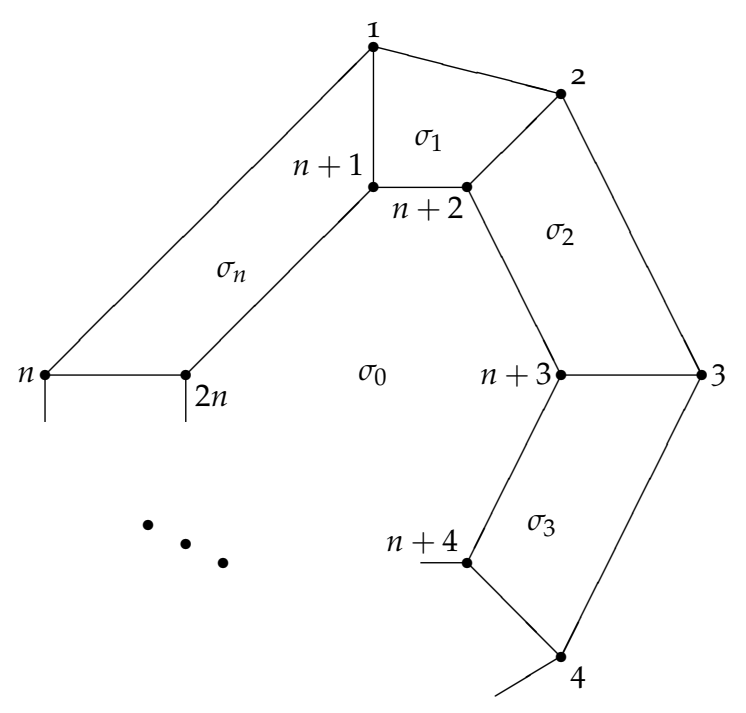

Figure 2. $\operatorname{cube}(\sigma)$

rows and $2 n-3$ columns. Call this matrix $S$. The first $n$ columns correspond to the cones $\sigma_{1}, \ldots, \sigma_{n}$. The last $n-3$ columns correspond to the cone $\sigma_{0}$. Each column has exactly four non-zero entries corresponding to the linear dependence relation among four vectors in $\mathbb{R}^{3}$. Upon normalization, one of the four numbers in each column can be chosen to be 1 . The matrix $S$ looks like

$$
S=\left[\begin{array}{ccccccccc}
a_{1} & 0 & 0 & & b_{n} & x_{4} & x_{5} & & x_{n} \\
b_{1} & a_{2} & 0 & & 0 & y_{4} & y_{5} & & y_{n} \\
0 & b_{2} & a_{3} & \ldots & 0 & z_{4} & z_{5} & \ldots & z_{n} \\
0 & 0 & b_{3} & & 0 & 1 & 0 & & 0 \\
0 & 0 & 0 & & 0 & 0 & 1 & & 0 \\
& & & \vdots & & & & \vdots & \\
0 & 0 & 0 & & a_{n} & 0 & 0 & & 1 \\
1 & 0 & 0 & \ldots & c_{n} & 0 & 0 & \ldots & 0 \\
c_{1} & 1 & 0 & & 0 & 0 & 0 & & 0 \\
0 & c_{2} & 1 & & 0 & 0 & 0 & & 0 \\
0 & 0 & c_{3} & & 0 & 0 & 0 & & 0 \\
& & & \vdots & & & & \vdots & \\
0 & 0 & 0 & \ldots & 1 & 0 & 0 & & 0
\end{array}\right]
$$

In $S$, rows 4 through $n$ are independent. The lower left $n \times n$ block of $S$ has determinant $1+(-1)^{n-1} c_{1} c_{2} \cdots c_{n}$ which is non-zero for a general cubing. Therefore, we see that $S$ has row rank $2 n-3$ and the kernel of $S$, which is $\operatorname{SF}(\Xi)$, has dimension three. 


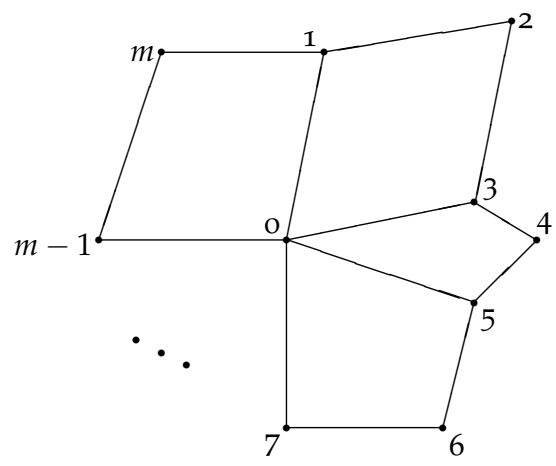

FigURE 3. $\operatorname{Star}\left(r_{0}\right)$

Lemma 4. Let $\Delta$ be a fan on $\mathbb{R}^{3}$ and $\sigma \in \Delta(3)$. Let $\Sigma$ be the subdivision of $\Delta$ corresponding to a cubing of $\sigma$. For a sufficiently general choice of $r_{n+1}, \ldots, r_{2 n}$ in the cubing, the natural map $\mathrm{SF}(\Delta) \rightarrow \mathrm{SF}(\Sigma)$ is an isomorphism.

Proof. As was shown in Lemma 3, any support function $h$ on a general cube $(\sigma)$ is linear. So given $h \in \mathrm{SF}(\Sigma), h$ is linear on the subfan $\left\{0, r_{1}, \ldots, r_{2 n}\right\}$. Thus, $h$ comes from a $\Delta$-linear support function.

Without loss of generality, fix $B$ and suppose $\Delta$ is a general member of $B$. Let $r_{0} \in \Delta(1)$. Define

$$
\operatorname{Star}\left(r_{0}\right)=\left\{\sigma \in \Delta \mid r_{0} \text { is a face of } \sigma \text { or } \sigma \text { is a face of } r_{0}\right\} \text {. }
$$

So $\operatorname{Star}\left(r_{0}\right)$ is a subfan of $\Delta$.

Lemma 5. If $h \in \operatorname{SF}(\Delta)$ is non-linear on $\Delta$, then there exists $r_{0} \in \Delta(1)$ such that $h$ is non-linear on $\operatorname{Star}\left(r_{0}\right)$.

Proof. Pick an arbitrary $\sigma \in \Delta(3)$. Subtract a linear support function from $h$ and assume without loss of generality that $h$ is zero valued on $\sigma$. Since $h$ is nonlinear, there exists some three-dimensional cone in $\Delta(3)$ on which $h$ is non-zero. Therefore, there are two three-dimensional cones $\sigma_{1}$ and $\sigma_{2}$ such that $\sigma_{1} \cap \sigma_{2}$ has dimension at least one and $h$ is zero on $\sigma_{1}$ and non-zero on $\sigma_{2}$. Let $r_{0}$ be a onedimensional face of $\sigma_{1} \cap \sigma_{2}$.

Say $\operatorname{Star}\left(r_{0}\right)(1)=\left\{r_{0}, r_{1}, \ldots, r_{m}\right\}$, as shown in Figure 3 . Check that $r_{0}, r_{1}, \ldots, r_{m}$ are distinct. Let $\Sigma \rightarrow \Delta$ be a subdivision of $\Delta$ corresponding to cubing every $\sigma$ in $\Delta(3)-\operatorname{Star}\left(r_{0}\right)(3)$. By Lemma 4 , we can pick $\Sigma$ such that the natural map $\mathrm{SF}(\Delta) \rightarrow \mathrm{SF}(\Sigma)$ is an isomorphism.

Lemma 6. For every $h \in \mathrm{SF}(\Sigma)$, the values of $h$ on $r_{0}, r_{1}, \ldots, r_{m}$ are determined by the values of $h$ on $\Sigma(1)-\left\{r_{0}, r_{1}, \ldots, r_{m}\right\}$.

Proof. The subdivision construction employed in the definition of $\Sigma$ guarantees that for each $i$ in the range $1 \ldots m$, there is a three-dimensional cone $\sigma \in \Sigma(3)$, $\sigma \notin \operatorname{Star}\left(r_{0}\right)$, such that $r_{i}$ is an edge of $\sigma$ and the other three edges of $\sigma$ are in $\Sigma(1)-\left\{r_{0}, r_{1}, \ldots, r_{m}\right\}$. The value of $h$ on $r_{i}$ is completely determined by the values on the three edges of $\sigma$ not in $\operatorname{Star}\left(r_{0}\right)$. 
Lemma 7. Every $\Sigma$-linear support function is linear on $\operatorname{Star}\left(r_{0}\right)$.

Proof. Any $\Sigma$-linear support function is determined by the values along the onedimensional cones of $\Sigma$. By this, there is an embedding

$$
0 \rightarrow \mathrm{SF}(\Sigma) \rightarrow \coprod_{r \in \Sigma(1)} \mathbb{R} \cdot r
$$

Let $h$ be a $\Sigma$-linear support function. The value of $h$ along $r_{0}$ is determined by the values along those one-dimensional cones not in $\operatorname{Star}\left(r_{0}\right)$. Therefore (6) yields an embedding

$$
0 \rightarrow \mathrm{SF}(\Sigma) \rightarrow \coprod_{r \in \Sigma(1)-\operatorname{Star}\left(r_{0}\right)} \mathbb{R} \cdot r
$$

View $r_{0}$ as a variable which parametrizes fans in an open neighborhood of $\Sigma$. For each such $r_{0}$, the group of support functions remains constant when viewed as a subspace of $\bigsqcup_{r \in \Sigma(1)-S \operatorname{Star}\left(r_{0}\right)} \mathbb{R} \cdot r$. Say $\sigma_{1}$ and $\sigma_{2}$ are adjacent three-dimensional cones in $\operatorname{Star}\left(r_{0}\right)$. Say $\sigma_{1}$ is spanned by $r_{0}, r_{1}, r_{2}, \ldots, r_{u}$ and $\sigma_{2}$ is spanned by $r_{0}, r_{1}, s_{2}, \ldots, s_{v}$. Fix $h \in \mathrm{SF}(\Sigma)$. Subtract a linear support function, and assume $h$ is zero valued on the cone $\sigma_{1}$. Because $\Delta$ is general, we can assume $r_{0}$ is general. Moving $r_{0}$ to a one-dimensional cone in the interior of $\sigma_{2}$ shows that $h$ is zero valued on a three-dimensional subset of $\sigma_{2}$. Hence $h$ is zero valued on $r_{1}, s_{2}, \ldots, s_{v}$. By iterating this argument, we see that $h$ is zero valued on $r_{1}, r_{2}, \ldots, r_{m}$. This proves the lemma.

Since $\operatorname{SF}(\Delta) \rightarrow \operatorname{SF}(\Sigma)$ is an isomorphism, it follows from Lemma 7 that every $\Delta$-linear support function is linear on $\operatorname{Star}\left(r_{0}\right)$. Theorem 1 follows from Lemma 5 .

\section{Concluding Remarks}

We conclude with some corollaries to Theorem 1 .

Corollary 1. Let $\Delta$ and $B$ be as in Theorem 1 . There is a dense open $U \subseteq B$ such that for all fans $\Delta^{\prime}$ in $U \cap B_{\text {rat, }}$

(1) the Picard group of $T_{N} \mathrm{emb}\left(\Delta^{\prime}\right)$ is zero

(2) $T_{N} \operatorname{emb}\left(\Delta^{\prime}\right)$ is non-projective.

(3) $\Delta^{\prime}$ is not the fan of cones over the faces of any convex integral polytope containing the origin in its interior.

Proof. As was shown in [5, p. 4044], there is an open subset $U$ of $B$ from which $\Delta^{\prime}$ may be chosen. In fact $U$ is the complement of a Zariski closed. It follows from Theorem 1 that there is a dense open $U \subseteq B$ such that for all rational fans $\Delta^{\prime}$ in $U$, the Picard group of $T_{N} \mathrm{emb}\left(\Delta^{\prime}\right)$ is zero.

A projective normal variety $X$ will always have a non-principal Cartier divisor corresponding to a hyperplane section. It follows that a projective toric variety has a non-zero Picard group. Therefore, for all rational fans $\Delta^{\prime}$ in $U$, the toric variety $T_{N} \mathrm{emb}\left(\Delta^{\prime}\right)$ is non-projective.

If $Q$ is a convex integral polytope containing the origin in its interior, and $\Delta^{\prime}$ the fan over the faces of $Q$, then $T_{N} \operatorname{emb}\left(\Delta^{\prime}\right)$ is projective [7, Proposition 2.19]. 
Remark 2. The proof of [3, Theorem 3.1] shows that for any complete fan of dimension three there exists a rational fan $\Sigma$ of the same combinatorial type as $\Delta$ such that $X=T_{N} \operatorname{emb}(\Sigma)$ is projective and thus Pic $X \neq 0$. Can $\Sigma$ be chosen from within $B$ ?

Theorem 1 also gives information about the rank of the torsion free part of the cohomological Brauer group [2] for a general fan $\Delta^{\prime}$ in $B$.

Corollary 3. Let $\Delta, B, U$ be as in Corollary 1. For all fans $\Delta^{\prime}$ in $U \cap B_{\text {rat }}$,

$$
\operatorname{dim} \mathrm{H}^{2}\left(T_{N} \operatorname{emb}\left(\Delta^{\prime}\right), \mathrm{G}_{m}\right) \otimes \mathbb{Q}=3-(n+1)+\sum_{i=0}^{w} q_{i}
$$

where $\Delta(3)=\left\{\sigma_{0}, \ldots, \sigma_{w}\right\}, \Delta(1)=\left\{r_{0}, \ldots, r_{n}\right\}$ and $q_{i}+3$ is the number of onedimensional edges in $\sigma_{i}$.

Proof. Follows from Corollary 1 and [5, Theorem 3.5].

Remark 4. It follows from Theorem 1 that Conjecture 4.15 of [4] is true.

\section{REFERENCES}

[1] V. I. Danilov, The geometry of toric varieties, Uspekhi Mat. Nauk 33 (1978), no. 2(200), 85-134, 247. MR 80g:14001

[2] F. R. DeMeyer, T. J. Ford, and R. Miranda, The cohomological Brauer group of a toric variety, J. Algebraic Geom. 2 (1993), no. 1, 137-154. MR 93i:14051

[3] Markus Eikelberg, Picard groups of compact toric varieties and combinatorial classes of fans, Results Math. 23 (1993), no. 3-4, 251-293. MR 94b:14052

[4] T. J. Ford, The toroidal embedding arising from an irrational fan, Results Math. 35 (1999), no. 1-2, 44-69. MR 2000a:14062

[5] Timothy J. Ford, Topological invariants of a fan associated to a toric variety, Comm. Algebra 23 (1995), no. 11, 4031-4045. MR 96g:14043

[6] William Fulton, Introduction to toric varieties, Princeton University Press, Princeton, NJ, 1993. MR 94g:14028

[7] Tadao Oda, Convex bodies and algebraic geometry, Springer-Verlag, Berlin, 1988. MR 88m:14038

Department of Mathematics, Florida Atlantic University, Boca Raton, Florida 33431

E-mail address: ford@fau.edu

Department of Mathematics, florida Atlantic University, Boca Raton, Florida 33431.

Current address: Commandant, ATSA-CDW, 5800 Carter Road, Fort Bliss, Texas 79916

E-mail address: rstimets@netscape.net 